\title{
SMARThivCD4mos: A complexity-free and cost effective model technology for monitoring HIV patients CD4 number in resource-poor settings
}

\author{
Aboubakar Yari ${ }^{1}{ }^{*}$, Frederic S. Passo ${ }^{1}$, Venus Yari $^{1}$, Ousmane Sanni ${ }^{1}$, Myra Yari $^{1}$, Mathieu Z. Dovenou ${ }^{1}$, Rachide Traore $^{1}$ \\ and Jean-Pierre Hounyet ${ }^{2}$ \\ ${ }^{1}$ Biotech tropicana, Inc, Parakou, Benin; ${ }^{2}$ Hôpital Saint-Jean de Dieu, Boko, Benin ; Aboubakar Yari* - E-mail: ayari@biotechtropicana.com; * \\ Corresponding author
}

received December 03, 2007; revised January 12, 2008; accepted January 19, 2008; published January 27, 2008

\begin{abstract}
:
We design a "simple" and "low cost" model technology for monitoring HIV patients CD4 number in resource-poor settings: SMARThivCD4mos. Cost and complexity are the major challenges to the developing world in transferring and implementing HIV surveillance technologies. We previously proposed a "three tests" combo kit model for improving HIV patients monitoring standards in resource-poor settings. From the pool of recommended alternative CD4 counting technologies, our "three tests" combo kit model retained the Capcellia technology for its "simplicity". However, compared to other CD4 counting technologies, such as Dynabeads, the Capcellia technology is "expensive”. Here, we describe a cost reduction strategy of the Capcellia technology.
\end{abstract}

Keywords: CD4 count; HIV surveillance technologies; cost and complexity; resource-poor countries; alternative technologies

Background:

CD4 number is a marker used in the clinical management of HIV patients to make important decisions including classifying diseases stages, monitoring disease progression, and guiding initiation of AntiRetroViral (ARV) treatment. CD8 cells are a subset of immune cells different from CD4 that are used as marker in monitoring other immune disorders, but not recommended in the World Health Organisation guidelines for the clinical management of HIV patients. [1, 2]

Flow cytometry is the standard technology for CD4 counting. However, the technology is complex and expensive, and requires a high level expertise for implementation, thereby limiting its widespread use in resource-poor settings. [1, 2] Resources and expertise are scarce in the developing world. $[1,2,3]$

Expert panels recommended cheaper and simpler so called alternative technologies, for the clinical management of HIV patients in the developing world. A number of simpler and cheaper CD4 counting technologies amenable to resourcepoor setting, have been developed. [1, 2, 3]

The CD4 counting Dynabeads technology showed a promise as an alternative to the standard flow cytometry because of its good correlation with the standard flow cytometry. However, the technology is complex. The International Medical Center of Japan developed a strategy to reduce the complexity and the cost of the Dynabeads technology, thereby permitting its widespread use in resource-limited situations. [1]

In our SMARThivPack model, we have previously demonstrated that cost and complexity of HIV surveillance technologies may be reduced, not only at a first technology development level, but also at a second technology implementation, and a third global coordination levels. Also, ISSN 0973-2063

Bioinformation 2(6): 257-259 (2008) our SMARThivPack model favours "automation" over "manual" text execution, and "three tests" over a "single test" in the clinical management of HIV patients in resource-poor settings. [3]

The Capcellia technology is an elisa-based CD4 counting system. CD4 counting is performed by the "automated" elisa plate reader. Our SMARThivPack model determined the Capcellia technology as "simple". However, the Capcellia technology is "expensive". [2, 3] compared to the \$3/test using the International Medical Center of Japan "Modified Dynabeads" protocol, CD4 counting using the Capcellia technology is more than 5 times at \$17/test. $[\mathbf{1}, \mathbf{3}]$

We hypothesize that, by applying the strategy developed at the International Medical Center of Japan to reduce cost of the "complex" Dynabeads technology, the cost of the "simple" but "expensive" Capcellia technology may be reduced, thereby combining "simplicity" with "low cost" within a "single technology body": SMARThivCD4mos.

\section{Description:}

We used web-based data mining and detailed investigation of existing CD4 counting protocols, to design a model technology for monitoring CD4 number in resource-poor settings. Using our cost and complexity level standards for guidance, we aim to design a CD4 counting system that is compatible with our SMARThivPack model. [3] Our cost standard favours "low cost" over "high cost", and our complexity level standard favours "user friendly" "automated" text execution, over "manual" test execution. Also, our SMARThivPack model favours specialisation to a limited number of technologies, over technology and skill diversity. In modelling our SMARThivCD4mos we hypothesize that, application of the International Medical 
Center of Japan cost reduction strategy of the complex CD4 Dynabeads technology, to the simple but expensive Capcellia technology, could yield a new technology that is both simple and cheap (Figure 1), and compatible with our
SMARThivPack Model. We modelled a first technology development level and a second technology implementation cost reductions strategies.

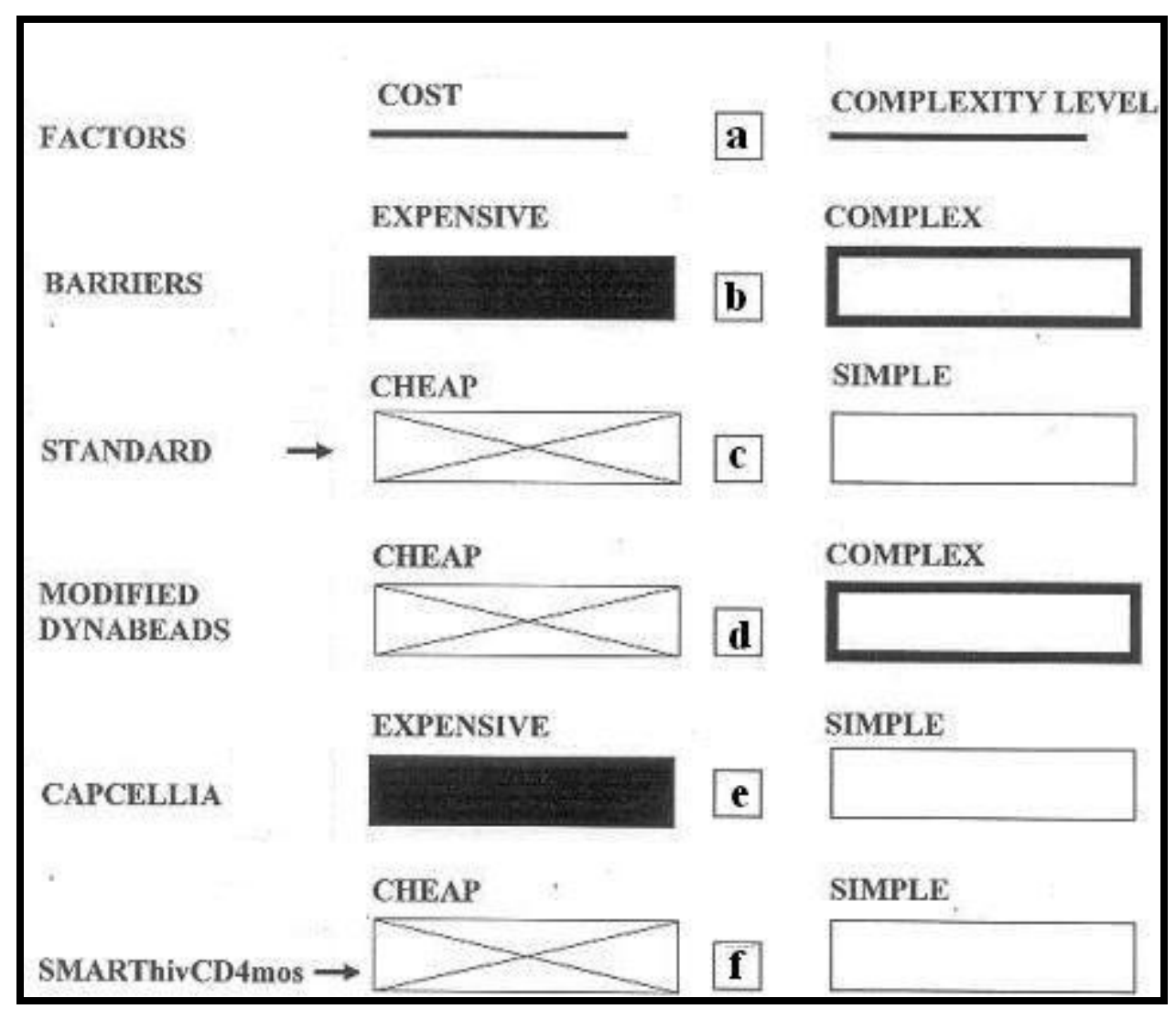

Figure 1: Comparison of cost and complexity factors (a) of SMARThivCD4mos with the Modified Dynabeads and the Capcellia technologies. Lack of resources and expertise are barriers to transfer of expensive and complex technologies to the developing world. (b) Cheap and simple technologies are the standards for the developing world (c) The modified Dynabeads technology is cheap but complex (d) while the Capcellia technology is simple but expensive (f) SMARThivCD4mos is both cheap and simple

At the first technology development level, we eliminate the CD8 component of the Capcellia kit. Relying on the World Health Organisation (WHO) guidelines recommending CD4 and not CD8 counting in the clinical management of HIV patients, the imjc eliminated CD8 counting components of the original Dynabeads protocol [1]. We applied the same principle to eliminate the CD8 component of the Capcellia kit. However, while the imcj reduced cost of the Dynabeads technology at the second implementation level through test execution protocol modification, our model reduces cost of the Capcellia technology at a first technology development level by eliminating CD8 counting components of the Capcellia kit. In the original Dynabeads protocol CD8 counting is a "binding" step. Execution of the original protocol commits to counting CD4 cells AND CD8 cells. In the Capcellia technology, CD8 counting is optional. The Capcellia protocol is constructed with flexibility permitting text execution design for counting only "CD4" cells", OR counting only "CD8 cells". However, the Capcellia kit comports both essential components for CD4 and CD8 counting. The Capcellia kit may be used not only in the clinical management of HIV patients, but also in monitoring other immune disorders; e.g. after an organ transplant. [2] Our SMARThivPack model is designed only for the clinical ISSN 0973-2063 Bioinformation 2(6): 257-259 (2008) management of HIV patients. Because, the World Health Organisation guidelines recommended CD4 counting and not CD8 counting in the clinical management of HIV patients, the CD8 counting components of the Capcellia kits are therefore not essential in the clinical management of HIV patients. Therefore, the CD8 counting components are unnecessary raising the cost of the Capcellia kit. To reduce cost, our SMARThiv CD4mos model eliminates the CD8 counting component of the Capcellia kit at the first technology development level.

At a second technology implementation level, we modelled a cost reduction strategy of the Capcellia technology that modified the Capcellia test execution protocol. Both the Dynabeads and the Capcellia test execution protocols comport a sample preparation step involving T-cells separation from whole blood, using magnetic beads. [1, 2] The International Medical Center of Japan "Modified Dynabeads" protocol reduced cost of the Dynabeads technology by reducing the quantity of required beads for T-cell separation. Our SMARThivCD4mos model hypothesizes that the cost of the Capcellia technology may be similarly reduced by reducing the quantity of required beads for T-cell separation. Detailed investigation of the Capcellia protocol and the "Modified 


\section{Bioinformation}

Dynabeads” protocol is in progress at Biotech tropicana, Inc to isolate additional modifiable targets for cost reduction of the "simple" but "expensive" Capcellia technology.

SMARThivCD4mos is compatible with our SMARThivPack "three tests" combo kit model. Compared to the original Dynabeads protocol, the International Medical Center of Japan modified protocol is both cheaper and simpler. [1] However, our SMARThivPack model previously determined the Dynabeads technology as complex. [3] The International Medical Center of Japan complexity reduction of the Dynabeads technology does not still overcome our complexity level standard. The Dynabeads technology relied on "manual counting" of CD4 cells. Furthermore, the use of microscope required a different skill from the skills required in elisa assays preferred in our SMARThivPack model. Based on the complexity level standard in our SMARThivPack model, "automation" should always be favoured over "manual execution" whenever possible. In the elisa-based Capcellia technology, CD4 counting is automated. However, the Capcellia technology is expensive. Compared to the \$3/test using the International Medical Center of Japan "Modified Dynabeads", [1] the cost/test using the simple Capcellia technology is more than 5 times at $\$ 17 /$ test. [3] Our hypothetical two levels modifications would reduce the cost/test of the "simple" Capcellia technology. The automated elisa plate reader counting of the CD4 cells makes the Capcellia technology compatible with our SMARThivPack model, thereby promoting "three tests" over "single test" in the clinical management of HIV patients, in resource-poor settings.

Capcellia is an old technology developed in the 1990s. The world Health Organisation (WHO) previously declassified the older Capcellia technology as "obsolete", and classified the newer Dynabeads technology in the pool of recommended technologies for the developing world. However, based on our standard favouring "automation" over, "human execution", we determined that the old "simple" Capcellia technology is better suited than the new "complex" Dynabeads technology for monitoring HIV patients in resource-poor settings. Furthermore, the International Medical Center of Japan determined the Dynabeads technology "too complex" to justify its modification of the original Dynabeads protocol.

SMARThivCD4mos is an intended component of the Biotech tropicana, Inc SMARThivPack. [3] SMARThivPack is an intended component of the Biotech tropican, Inc "Life Box" comporting in addition to the SMARThivPack "three tests" Combo kit, an elisa plate reader and a PCR set up.

The Biotech tropicana, Inc "Life Box" is an intended component of the Biotech tropicana, Inc SMARThivGLOBALmos, a third level global coordination cost and complexity reduction model comporting in addition to the SMARThivPack-based "Life Box", alternative life boxes ("A-Life Box") designed based on existing equipments in a specific laboratory to overcome cost and complexity.

Overall, applying a cost reduction strategy developed at the International Medical Center of Japan, to reduce cost of the "complex" Dynabeads technology, we modelled a cost reduction strategy of the "simple" Capcellia technology, and design a new model technology that combines "simplicity" and "low cost" within a "single technology body": SMARThivCD4mos. SMARThivCD4mos is compatible with our SMARThivPack model for improving HIV patients monitoring standards in resource-poor settings.

\section{References:}

[01] B. Xiuqiong, et al., J Acquir Immune Defic Syndr., 38: 1 (2005) [PMID: 15608516]

[02] D. Carriere, et al., Clin Chem., 45: 92 (1999) [PMID: 9895343]

[03] A. Yari, et al., Bioinformation, 2: 97 (2007)

Edited by P. Kangueane Citation: Yari et al., Bioinformation 2(6): 257-259 (2008) License statement: This is an open-access article, which permits unrestricted use, distribution, and reproduction in any medium, for non-commercial purposes, provided the original author and source are credited. 